\title{
Drug-Coated Balloon-Only Angioplasty Outcomes in Diabetic and Nondiabetic Patients with De Novo Small Coronary Vessels Disease
}

\author{
Botey Katamu Benjamin (iD, ${ }^{1}$ Wenjie Lu, ${ }^{1}$ Zhanying Han, ${ }^{1}$ Liang Pan, ${ }^{1}$ Xi Wang, \\ Xiaofei Qin, ${ }^{1}$ Guoju Sun, ${ }^{1}$ Xule Wang, ${ }^{1}$ Yingguang Shan, ${ }^{1}$ Ran Li, ${ }^{1}$ Xiaolin Zheng, \\ Wencai Zhang, ${ }^{1}$ Qiangwei Shi, ${ }^{1}$ Shuai Zhou, ${ }^{1}$ Sen Guo, ${ }^{1}$ Peng Qin, ${ }^{2}$ Chhatra Pratap Singh, \\ Jianzeng Dong $\mathbb{D}^{1},{ }^{1}$ and Chunguang Qiu $\mathbb{D}^{1}$ \\ ${ }^{1}$ Department of Cardiovascular Medicine, The First Affiliated Hospital of Zhengzhou University, Zhengzhou, China \\ ${ }^{2}$ Department of Geriatric Cardiology, The First Affiliated Hospital of Zhengzhou University, Zhengzhou, China
}

Correspondence should be addressed to Chunguang Qiu; qcg123@163.com

Received 11 July 2021; Accepted 18 November 2021; Published 1 December 2021

Academic Editor: Thach N. Nguyen

Copyright (C) 2021 Botey Katamu Benjamin et al. This is an open access article distributed under the Creative Commons Attribution License, which permits unrestricted use, distribution, and reproduction in any medium, provided the original work is properly cited.

\begin{abstract}
Background. The revascularization of small vessels using drug-eluting stents remains challenging. The use of the drug-coated balloon is an attractive therapeutic strategy in de novo lesions in small coronary vessels, particularly in the diabetic group. This study aimed to assess the outcomes of DCB-only angioplasty in small vessel disease. Methods. A total of 1198 patients with small vessel disease treated with DCB-only strategy were followed. Patients were divided into the diabetic and nondiabetic groups. Clinical and angiographical follow-up were organized at 12 months. The primary endpoints were target lesion failure and secondary major adverse cardiac events. Results. There was a significantly higher rate of target lesion failure among diabetic patients compared to nondiabetic [17 (3.9\%) vs. $11(1.4 \%), P=0.006]$, taken separately, the rate of target lesion revascularization significantly differed between groups with a higher rate observed in the diabetic group [9 (2\%) vs. $4(0.5 \%), P=0.014]$. Diabetes mellitus remained an independent predictor for TLF (HR: 2.712, CI: 1.254-5.864, $P=0.011$ ) and target lesion revascularization (HR: 3.698, CI: 1.112-12.298, $P=0.033$ ) after adjustment. However, no significant differences were observed between groups regarding the target vessel myocardial infarction $(0.6 \%$ vs. $0.1 \%, P=0.110)$ and MACE $[19(4.4 \%)$ vs. $21(2.7 \%), P=0.120]$. Conclusion. Drug-coated balloon-only treatment achieved lower incidence rates of TLF and MACE. Diabetes is an independent predictor for target lesion failure and target lesion revascularization at one year following DCB treatment in small coronary vessels. We observed no significant differences between groups regarding MACE in one year.
\end{abstract}

\section{Introduction}

Small vessel coronary disease is an independent predictor for poor outcomes after the percutaneous coronary intervention (PCI) and accounts each year for 30 to $50 \%$ of all-comers coronary intervention procedures worldwide [1-4]. The best treatment strategy for small vessel coronary disease remains a real challenge [5]. Small vessel disease (SVD) poses concrete limitations to the use of drug-eluting stents (DES); the implantation of DES in small vessels is confronted with higher rates of stent failure, restenosis, and repeat revascularization consecutive to the late lumen loss (LLL) [3-8]. This is partly related to the fact that DES leave permanent metallic struts and polymeric matrix within treated vessels which behave as persistent inflammatory stimulus and hinder the full restoration of vessels endothelial functions and the capacity to increase lumen diameter over time $[9,10]$. The accelerated LLL is related to the small vessel's restricted capability to accommodate to even small neointimal proliferation following stent implantation; that is, for 
a given neointimal proliferation, the relative lumen reduction will be more pronounced in the small vessel as compared to big vessel $[1,11-13]$. Besides, there is an established association between small vessel coronary disease with diabetes mellitus, which is also a powerful predictor for poor outcomes after PCI [1, 14-20]. Conceived to overcome the drawbacks observed with DES, emerging evidence suggests using drug-coating balloons (DCB) as an interesting alternative treatment strategy in small vessel disease [21-25]. Because of the higher prevalence of SVD observed in diabetic patients, this group of patients is more likely to be treated with the drug-coated balloon. There are no available data comparing DCB outcomes in diabetic and nondiabetic patients with SVD to the best of our knowledge. It is not clear whether treatment strategy with DCB in this particular setting will result in the same trend of outcomes as observed when DES are used provided that DCB fulfill therapeutic goals of DES without reproducing its limitations and drawbacks. Therefore, this study aims to assess diabetic and nondiabetic patients' outcomes after drug-coated balloononly strategy in de novo small vessel coronary disease.

\section{Material and Methods}

2.1. Study Design and Population. This is a prospective, single-center, observational study of 1198 patients undergoing PCI for de novo lesion in the native small coronary vessels with DCB as the only therapeutic strategy. We defined small vessel disease as reference vessel diameter $\leq 2.75 \mathrm{~mm}$. Based on the presence of diabetes, patients were divided into the diabetic and nondiabetic groups. We considered as diabetic patients with fasting plasma glucose $\geq 126 \mathrm{mg} / \mathrm{dL}(7.0 \mathrm{mmol} / \mathrm{L})$ and/or two-hour plasma glucose $\geq 200 \mathrm{mg} / \mathrm{dL}(11.1 \mathrm{mmol} / \mathrm{L})$ during an oral glucose tolerance test and/or $\mathrm{HbAlc} \geq 6.5 \%$ [26]. Clinical follow-up was conducted at 12-month postprocedure or at any time when needed.

2.2. Exclusion Criteria. A larger vessel with $>2.75 \mathrm{~mm}$ diameter, in-stent restenosis, hybrid treatment of the target lesion, simultaneous treatment of small and large vessels lesions, simultaneous treatment of in-stent restenosis and de novo lesions, renal failure requiring dialysis, contraindication to dual antiplatelet therapy, and bailout stent implantation.

2.3. Study Endpoints and Definition. The primary endpoint of the study were target lesion failure (TLF) defined as the composite of ischemia-driven revascularization of the target lesion, myocardial infarction related to the target vessel, or cardiac death related to the target vessel. Whenever it was not possible to determine with certainty whether the myocardial infarction or death was related to the target vessel, it was considered a TLF. The secondary endpoints were major adverse cardiac events (MACE) defined as the composite of all-cause death, myocardial infarction, and target vessel revascularization (TVR). We considered as clinically driven target lesion revascularization (TLR) any revascularization of the target lesion performed due to a stenosis $>50 \%$ and either evidence of clinical or functional ischemia or recurrence of the clinical syndrome for which the initial procedure was performed [27]. TLR was defined as any repeat percutaneous coronary intervention of the target lesion (including $10 \mathrm{~mm}$ proximal and $10 \mathrm{~mm}$ distal to the index device) or bypass surgery of the target vessel motivated by restenosis or other complications [27]. Target vessel myocardial infarction was defined as any myocardial infarction related to the target vessel or when not clearly related to any other vessel [27]. The diagnosis of myocardial infarction was made when the following criteria were met: detection of increase and or decrease of cardiac biomarkers levels; at least one value above the 99th percentile of the upper reference limit, together with evidence of myocardial ischemia, as recognized by at least one of the following: symptoms of ischemia, electrocardiogram changes of new ischemia or development of pathologic Q waves, and imaging evidence of new loss of viable myocardium or new regional wall motion abnormality [28]. Cardiac death was defined as death resulting from cardiovascular causes such as death caused by acute myocardial infarction, sudden cardiac death, including unwitnessed death, heart failure, stroke, cardiovascular procedures, cardiovascular hemorrhage, and death resulting from other cardiovascular causes [27]. We considered all-cause death as death related to any possible cause and TVR as any repeat percutaneous intervention or surgical bypass of any segment of the target vessel.

2.4. Interventions. We included patients whose interventions and postprocedure period were managed in line with our established criteria. Patients received oral aspirin $300 \mathrm{mg}$ at least $24 \mathrm{~h}$ before the intervention, oral clopidogrel or ticagrelor at least six hours before the procedure, respectively, at $300 \mathrm{mg}$ and $180 \mathrm{mg}$ (except for stable patients on ongoing dual antiplatelet therapy since a certain time) and then maintained aspirin at $100 \mathrm{mg} /$ day, clopidogrel at $75 \mathrm{mg} /$ day, or ticagrelor $90 \mathrm{mg}$ twice daily. In patients with the acute coronary syndrome, a loading dose of aspirin $300 \mathrm{mg}$ and ticagrelor $180 \mathrm{mg}$ or clopidogrel $600 \mathrm{mg}$ were orally administered at the admission before the procedure. The choice between clopidogrel and ticagrelor was at the physician's discretion. Intravenous administration of unfractionated heparin at $100 \mathrm{IU} / \mathrm{kg}$ was done a few minutes before PCI and activated clotting time was maintained at 250 to $350 \mathrm{~s}$ throughout the intervention. Careful lesion preparation was observed before applying the DCB and balloons were not used for postdilation once the DCB was deployed; the choice of the appropriate technique for lesion preparation and balloon selection was at the operator's discretion. We regarded as successful intervention treatment all postprocedural visually estimated $\leq 30 \%$ residual stenosis after PCI. After the procedure, patients received a dual antiplatelet therapy (DAPT) regimen, including daily aspirin $100 \mathrm{mg}$ in association with clopidogrel $75 \mathrm{mg}$ or ticagrelor $90 \mathrm{mg}$ twice daily for at least 6 months. 
2.5. Device Description. All patients were treated with the SeQuent Please which is a paclitaxel-coated balloon catheter (B. Braun Melsungen, Germany). The paclitaxel is coated at a dose of $3 \mu \mathrm{g} / \mathrm{mm}^{2}$ balloon surface, a matrix coating made of paclitaxel plus hydrophilic spacer (iopromide). A minimum of 40 seconds inflation time is needed to allow sufficient drug to be released into the vessel wall; only $4.5 \%$ of the drug remains on the balloon [4].

2.6. Quantitative Coronary Angiography. Quantitative coronary angiography measurements were performed off-line using a validated edge detection system (QAngio XA V7.3, Medis Medical Imaging, Netherlands) at a core laboratory (The First Affiliated Hospital of Zhengzhou University, Zhengzhou, China) by a team of experienced personnel who were blinded.

2.7. Follow-Up. All patients were clinically followed up at 12 months (visits in the hospital and by telephone); we used a structured clinical questionnaire to assess medication, quality of life, and events. The angiographic follow-up was organized at 12 months or at any time when needed. Angiographic data were vouched by a blinded core lab, and an independent clinical committee adjudicated all the study endpoints.

2.8. Statistical Analysis. Categorical variables are expressed as frequencies and percentages while continuous variables are reported as mean \pm standard deviation. As appropriate comparisons between categorical variables were evaluated using a 2-tailed Fisher's exact test or Pearson's chi-squared test, continuous variables were compared using Student's $t$ test. To identify independent predictors of TLF and TLR, a Cox proportional hazard multivariate analysis was performed to calculate the hazard ratios (HR) with 95\% confidence intervals (CI). Statistical significance was accepted for bilateral $P<0.05$.

The summary of the method is presented in Figure 1.

\section{Results}

3.1. Patients Baseline Characteristics. From a total of 1198 patients, 831 were male, and 367 were female; 430 had diabetes, and 768 were nondiabetic. Diabetic patients were significantly older and had a significantly higher proportion of hypertension, hyperlipidemia, and renal failure compared to the nondiabetic. Data are presented in Table 1.

3.2. Baseline Lesions Characteristics. Of 1351 treated lesions, 483 were observed in the diabetic group versus 868 in the nondiabetic group. There were numerically higher proportion of bifurcation, ostial lesion, thrombosis, and diffused lesion in the diabetic group, without significant differences. There were a numerically higher proportion of chronic total occlusion, calcified lesions, and longer mean lesion length in the nondiabetic group compared to the diabetic. Data are presented in Table 2.
3.3. Procedure Characteristics. Comparable results were observed between groups in terms of the reference vessel diameter $(2.5 \pm 0.2$ vs. $2.5 \pm 0.8 ; P=0.366)$, DCB inflation pressure $(8.3 \pm 5.12 \mathrm{~atm}$ vs. $8.1 \pm 1.42 \mathrm{~atm} ; P=0.416)$, $\mathrm{DCB}$ inflation time $(59.9 \pm 3.76 \mathrm{~s}$ vs. $60.1 \pm 4.93 \mathrm{~s} ; P=0.474)$, and postprocedural mean diameter residual stenosis $(20.3 \pm 11.4$ vs. $19.3 \pm 11.6 ; P=0.145)$ in the diabetic and nondiabetic, respectively. Data are provided in Table 3.

3.4. Outcomes. Of 1198 enrolled patients, 535 (44.65\%) had angiographic follow-up at 1 year. We observed significant differences between the groups regarding minimum lumen diameter (MLD) $(1.89 \pm 0.53$ vs. $2 \pm 0.39 ; P=0.07)$, LLL $(0.17 \pm 0.37$ vs. $0.09 \pm 0.25 ; P=0.003)$, and binary restenosis $(4.8 \%$ vs. $1.1 \% ; P=0.008)$ in the diabetic and nondiabetic, respectively. There were significant differences between groups in terms of TLF; the higher incidence rates were observed in the diabetic group (3.9\% vs. $1.4 \% ; P=0.006)$. TLF components taken separately showed significantly higher rates of TLR in the diabetic group ( $2 \%$ vs. $0.5 \% ; P=0.014$ ). However, comparable results were observed between groups regarding the target vessel myocardial infarction $(0.6 \%$ vs. $0.1 \% ; P=0.110)$. There were no significant differences between the two groups in the $\operatorname{MACE}(4.4 \%$ vs. $2.7 \% ; P=0.120)$. Data are presented in Table 4 and Figures 2 and 3.

3.5. Independent Predictor of Target Lesion Failure and Target Lesion Revascularization. We performed the multivariate logistic regression analysis to identify independent predictors of TLF and TLR; diabetes remained an independent predictor for TLF and MACE after adjustment. Results are presented in Table 5.

\section{Discussion}

In keeping with the proposed appropriate cutoff to define small vessels, and with the majority of DCB studies on the small vessel that used $<2.8 \mathrm{~mm}$ as the cutoff point for small vessel definition, we defined small vessel as estimated reference vessel diameter $\leq 2.75 \mathrm{~mm}[4,7]$. Many trials have demonstrated the noninferiority and safety of DCB compared to DES in treating the small coronary vessel $[23,29,30]$. This is the first clinical trial to compare the DCB outcomes in diabetic and nondiabetic patients with de novo small coronary vessels. The main findings of this prospective single-center observational study indicate the significantly higher rates of TLF ( $3.9 \%$ vs. $1.4 \% ; P=0.006)$ and TLR ( $2 \%$ vs. $0.5 \% ; P=0.014)$ in the diabetic group. A higher MACE rate $(4.4 \%$ vs. $2.7 \%$; $P=0.120)$ was observed with diabetic arm without reaching statistical significance. Diabetes remained an independent predictor for TLF (HR: 2.712; CI: $1.254-5.864 ; P=0.011$ ) and TLR (HR: 3.698; CI: 1.112-12.298; $P=0.033$ ) after adjustment. Our findings are in keeping with the previous studies that reported acceptable low rates of TLF and MACE with DCB in the small vessel $[11,21,29,31-37]$. The PEPCAD I study reported 6.1\% MACE events' rates after 12-month follow-up; the BASKET-SMALL 2 trial observed 7.5\% MACE rates in the DCB arm $[23,31]$. The DCB angioplasty in elderly patients with SVD reported a 9- 


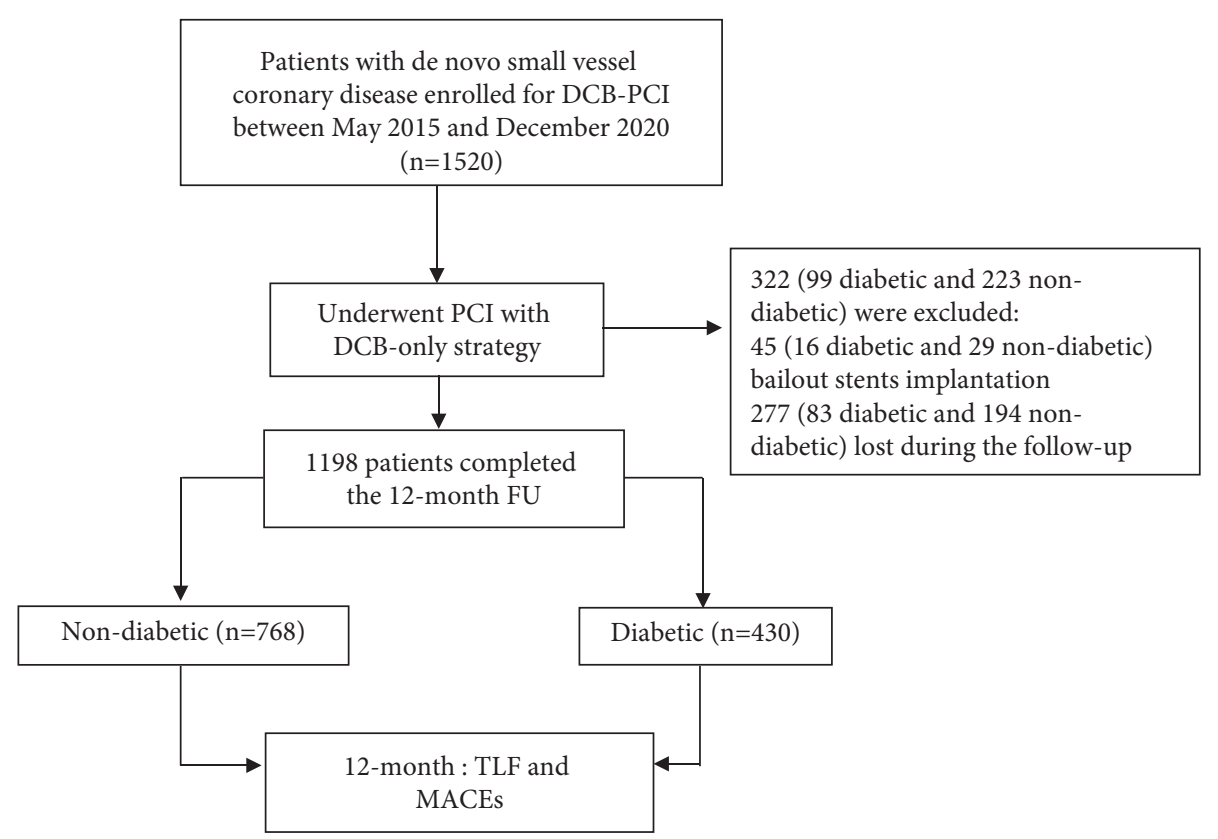

Figure 1: Study diagram. DCB: drug-coated balloon; FU: follow-up; MACEs: major adverse cardiac events; PCI: percutaneous coronary intervention; TLF: target lesion failure.

TABle 1: Patients' baseline characteristics.

\begin{tabular}{|c|c|c|c|c|}
\hline & Overall population $(n=1198)$ & Nondiabetic $(n=768)$ & Diabetic $(n=430)$ & $P$ value \\
\hline \multicolumn{5}{|l|}{ Demographics } \\
\hline \multicolumn{5}{|l|}{ Sex } \\
\hline Male & $831(69.3 \%)$ & $544(70.8 \%)$ & $287(66.7 \%)$ & 0.141 \\
\hline Female & $367(30.7 \%)$ & $224(29.1 \%)$ & $143(33.2 \%)$ & 0.141 \\
\hline Age & $60 \pm 10.7$ & $59 \pm 10.9$ & $60 \pm 10.5$ & $0.003^{*}$ \\
\hline \multicolumn{5}{|l|}{ History and risk factor } \\
\hline Family history of CAD & $223(18.6 \%)$ & $140(18.2 \%)$ & $83(19.3 \%)$ & 0.643 \\
\hline Current smoker & $380(31.7 \%)$ & $245(31.9 \%)$ & $135(31.3 \%)$ & 0.857 \\
\hline Hyperlipidemia & $340(28.3 \%)$ & $187(24.3 \%)$ & $153(35.5 \%)$ & $0.001^{*}$ \\
\hline History of MI & $117(9.7 \%)$ & $72(9.3 \%)$ & $45(10.4 \%)$ & 0.542 \\
\hline History of PCI & $191(15.9 \%)$ & $105(13.6 \%)$ & $86(20 \%)$ & $0.004^{*}$ \\
\hline History of CABG & $19(1.5 \%)$ & $9(1.1 \%)$ & $10(2.3 \%)$ & 0.125 \\
\hline \multicolumn{5}{|l|}{ Laboratory tests } \\
\hline Hemoglobin $(\mathrm{g} / \mathrm{L})$ & $135 \pm 62.4$ & $137 \pm 77.4$ & $131 \pm 15.2$ & 0.133 \\
\hline Hematocrit (\%) & $0.80 \pm 4$ & $0.94 \pm 4.7$ & $0.56 \pm 2.4$ & 0.141 \\
\hline Blood glucose $(\mathrm{mmol} / \mathrm{L})$ & $173 \pm 122$ & $145 \pm 91$ & $229 \pm 146$ & $0.001^{*}$ \\
\hline HbA1C (\%) & $6 \pm 3.4$ & $5 \pm 0.35$ & $8 \pm 5.06$ & $0.001^{*}$ \\
\hline Urea $(\mathrm{mmol} / \mathrm{dL})$ & $5 \pm 2.9$ & $5 \pm 1.8$ & $6 \pm 4.2$ & $0.003^{*}$ \\
\hline Creatinine $(\mu \mathrm{mol} / \mathrm{L})$ & $75 \pm 38.1$ & $75 \pm 34.9$ & $75 \pm 43$ & 0.960 \\
\hline eGRF & $90 \pm 17$ & $90 \pm 15.9$ & $89 \pm 18.7$ & 0.479 \\
\hline $\mathrm{LDL}(\mathrm{mmol} / \mathrm{dL})$ & $4 \pm 64.8$ & $5 \pm 82.5$ & $2 \pm 0.7$ & 0.424 \\
\hline $\mathrm{HDL}(\mathrm{mmol} / \mathrm{dL})$ & $2 \pm 31.7$ & $2 \pm 40.3$ & $0.9 \pm 0.2$ & 0.405 \\
\hline Albumin (g/L) & $10 \pm 13.5$ & $10 \pm 13.6$ & $10 \pm 13.4$ & 0.831 \\
\hline Total cholesterol $(\mathrm{mmol} / \mathrm{dL})$ & $3 \pm 2.5$ & $3 \pm 0.9$ & $3 \pm 3.9$ & 0.309 \\
\hline Triglyceride $(\mathrm{mmol} / \mathrm{dL})$ & $1 \pm 0.9$ & $1 \pm 0.9$ & $1 \pm 1.07$ & $0.001^{*}$ \\
\hline NT-proBNP (pg/ml) & $624 \pm 1049.2$ & $568 \pm 898.5$ & $720 \pm 1266.6$ & 0.079 \\
\hline $\mathrm{cTnT}(\mathrm{ng} / \mathrm{L})$ & $11 \pm 18.2$ & $11 \pm 18.2$ & $12 \pm 18.6$ & 0.560 \\
\hline cTnI (ng/L) & $1.68 \pm 3.8$ & $1 \pm 3.5$ & $1 \pm 4.3$ & 0.506 \\
\hline \multicolumn{5}{|l|}{ Clinical presentation } \\
\hline Hypertension & $623(52.3 \%)$ & $363(47.2 \%)$ & $260(60.4 \%)$ & $0.001^{*}$ \\
\hline LVEF (\%) & $24 \pm 16.6$ & $24 \pm 16.7$ & $25 \pm 16$ & 0.622 \\
\hline Acute coronary syndrome & $802(66.9 \%)$ & $515(67 \%)$ & $287(66.7 \%)$ & 0.912 \\
\hline Renal failure & $57(4.7 \%)$ & $25(3.2 \%)$ & $32(7.4 \%)$ & $0.001^{*}$ \\
\hline Insulin therapy & $72(6 \%)$ & $0(0 \%)$ & $72(16.7 \%)$ & 0.001 \\
\hline
\end{tabular}

${ }^{*}$ Statistically significant. CABG: coronary artery bypass graft; CAD: coronary artery disease; CRP: C-reactive protein; MI: myocardial infarction; cTnI: cardiac troponin I; cTnT: cardiac troponin T; eGFR: estimated glomerular filtration rate; HbA1C: glycosylated hemoglobin; HDL: high-density lipoprotein; LDL: lowdensity lipoprotein; NT-proBNP: N-terminal prohormone brain natriuretic peptide; PCI: percutaneous coronary intervention. 
TABLE 2: Baseline lesions characteristics.

\begin{tabular}{|c|c|c|c|c|}
\hline & Overall population $(n=1198)$ & Nondiabetic $(n=768)$ & Diabetic $(n=430)$ & $P$ value \\
\hline Bifurcation & $418(34.8 \%)$ & $259(33.7 \%)$ & $159(36.9 \%)$ & 0.257 \\
\hline Ostial lesion & $193(16.1 \%)$ & $121(15.7 \%)$ & $72(16.7 \%)$ & 0.655 \\
\hline Thrombosis & $7(0.5 \%)$ & $3(0.3 \%)$ & $4(0.9 \%)$ & 0.252 \\
\hline CTO & $181(15.1 \%)$ & $127(16.5 \%)$ & $54(12.5 \%)$ & 0.65 \\
\hline Diffusion lesion & $298(24.8 \%)$ & $188(24.4 \%)$ & $110(25.5 \%)$ & 0.672 \\
\hline Calcified & $61(5 \%)$ & $42(5.4 \%)$ & $19(4.4 \%)$ & 0.428 \\
\hline Target vessel & & & & 0.112 \\
\hline LAD & $505(42.2 \%)$ & $314(40.8 \%)$ & $191(44.4 \%)$ & 0.235 \\
\hline LCX & $456(38.1 \%)$ & $311(40.4 \%)$ & $145(33.7 \%)$ & $0.021^{*}$ \\
\hline RCA & $233(19.4 \%)$ & $140(18.2 \%)$ & $93(21.6 \%)$ & 0.154 \\
\hline Graft & $4(0.3 \%)$ & $3(0.3 \%)$ & $1(0.2 \%)$ & 0.640 \\
\hline Lesion length & $16 \pm 8.7$ & $16 \pm 8.6$ & $15 \pm 8.7$ & 0.644 \\
\hline Treated lesion & $1351(100 \%)$ & $868(64.2 \%)$ & $483(35.7 \%)$ & 0.064 \\
\hline 1 lesion & $1051(87.7 \%)$ & $674(87.7 \%)$ & $377(87.6 \%)$ & 0.965 \\
\hline 2 lesions & $141(11.8 \%)$ & $88(11.4 \%)$ & $53(12.3 \%)$ & 0.655 \\
\hline 3 lesions & $6(0.6 \%)$ & $6(0.7 \%)$ & $0(0 \%)$ & 0.066 \\
\hline
\end{tabular}

*Statistically significant; CTO: chronic total occlusion; LAD: left anterior descending artery; LCX: left circumflex; RCA: right coronary artery.

Table 3: Procedural characteristics.

\begin{tabular}{|c|c|c|c|c|}
\hline & Overall population $(n=1198)$ & Nondiabetic $(n=768)$ & Diabetic $(n=430)$ & $P$ value \\
\hline Procedural & & & & 0.225 \\
\hline Transradial & $1181(98.5 \%)$ & $736(95.8 \%)$ & $418(97.2 \%)$ & \\
\hline Transfemoral & $44(3.6 \%)$ & $32(4.1 \%)$ & $12(2.7 \%)$ & \\
\hline Treated lesion & $1351(100 \%)$ & $868(64.2 \%)$ & $483(35.7 \%)$ & 0.064 \\
\hline 1 lesion & $1051(87.7 \%)$ & $674(87.7 \%)$ & $377(87.6 \%)$ & \\
\hline 2 lesions & $141(11.8 \%)$ & $88(11.4 \%)$ & $53(12.3 \%)$ & \\
\hline 3 lesions & $6(0.6 \%)$ & $6(0.7 \%)$ & $0(0 \%)$ & \\
\hline \multicolumn{5}{|l|}{ Lesion preparation } \\
\hline Semicompliant balloon & $939(78.4 \%)$ & $609(79.2 \%)$ & $330(76.7 \%)$ & 0.303 \\
\hline Semicompliant balloon diameter $(\mathrm{mm})$ & $2 \pm 0.2$ & $2 \pm 0.2$ & $2 \pm 0.2$ & 0.647 \\
\hline Semicompliant balloon pressure (atm) & $8.2 \pm 3.2$ & $8.2 \pm 1.2$ & $8.3 \pm 3.6$ & 0.431 \\
\hline Lacrosse NSE ${ }^{\circledR}$ & $364(30.4 \%)$ & $295(38.4 \%)$ & $135(39.7 \%)$ & 0.569 \\
\hline Lacrosse $\mathrm{NSE}^{\circledR}$ diameter $(\mathrm{mm})$ & $2.3 \pm 0.2$ & $2.3 \pm 0.2$ & $2.3 \pm 0.2$ & 0.151 \\
\hline Cutting balloon & $130(10.9 \%)$ & $81(10.5 \%)$ & $49(11.3 \%)$ & 0.651 \\
\hline Cutting balloon diameter $(\mathrm{mm})$ & $2.5 \pm 0.2$ & $2.5 \pm 0.2$ & $2.5 \pm 0.2$ & 0.536 \\
\hline Scoreflex ${ }^{\circledR}$ & $51(4.3 \%)$ & $35(4.5 \%)$ & $16(3.7 \%)$ & 0.492 \\
\hline Scoreflex ${ }^{\circledR}$ diameter $(\mathrm{mm})$ & $2.2 \pm 0.2$ & $2.2 \pm 0.2$ & $2.2 \pm 0.2$ & 0.359 \\
\hline NC balloon & $207(17.3 \%)$ & $137(17.8 \%)$ & $70(16.7 \%)$ & 0.493 \\
\hline NC balloon diameter (mm) & $2.5 \pm 0.16$ & $2.5 \pm 0.17$ & $2.5 \pm 0.16$ & 0.861 \\
\hline NC balloon pressure (atm) & $14.3 \pm 2.4$ & $14.1 \pm 2.3$ & $14.4 \pm 3.5$ & 0.347 \\
\hline Rotational atherectomy & $26(2.2 \%)$ & $21(2.7 \%)$ & $5(1.1 \%)$ & 0.073 \\
\hline Burr diameter $(\mathrm{mm})$ & $1.4 \pm 0.1$ & $1.4 \pm 0.1$ & - & - \\
\hline Dissection after predilation & & & 0.540 & \\
\hline Type A & $377(31.4 \%)$ & $248(32.2 \%)$ & $129(30 \%)$ & 0.404 \\
\hline Type B & $159(13.2 \%)$ & $99(12.8 \%)$ & $60(13.9 \%)$ & 0.603 \\
\hline Residual stenosis after predilation & $21.1 \pm 11.2$ & $20.4 \pm 10.8$ & $22.2 \pm 11.8$ & $0.020^{*}$ \\
\hline Residual stenosis after DCB & $19.7 \pm 11.5$ & $19.3 \pm 11.6$ & $20.3 \pm 11.4$ & 0.145 \\
\hline DCB number & & & & 0.463 \\
\hline $1 \mathrm{DCB}$ & $1119(93.4 \%)$ & $716(93.2 \%)$ & $403(93.7 \%)$ & \\
\hline $2 \mathrm{DCB}$ & $76(6.3 \%)$ & $51(6.6 \%)$ & $25(5.8 \%)$ & \\
\hline $3 \mathrm{DCB}$ & $3(0.3 \%)$ & $1(0.13 \%)$ & $2(0.4 \%)$ & \\
\hline Stenosis (\%) & $88.9 \pm 9.4$ & $88.9 \pm 9.6$ & $88.8 \pm 9.1$ & 0.890 \\
\hline $\operatorname{RVD}(\mathrm{mm})$ & $2.5 \pm 0.7$ & $2.5 \pm 0.8$ & $2.5 \pm 0.2$ & 0.366 \\
\hline DCB diameter $(\mathrm{mm})$ & $2.4 \pm 0.2$ & $2.4 \pm 0.2$ & $2.4 \pm 0.2$ & 0.629 \\
\hline DCB length $(\mathrm{mm})$ & $23.2 \pm 9.3$ & $23.3 \pm 9.2$ & $23 \pm 9.4$ & 0.660 \\
\hline DCB inflation pressure (atm) & $8.2 \pm 3.2$ & $8.1 \pm 1.4$ & $8.3 \pm 5.1$ & 0.416 \\
\hline DCB inflation time (s) & $60 \pm 4.5$ & $60.1 \pm 4.9$ & $59.9 \pm 3.7$ & 0.474 \\
\hline Dissection after DCB deployment & & & 0.773 & \\
\hline Type A & $338(28.2 \%)$ & $218(28.3 \%)$ & $120(27.9 \%)$ & 0.860 \\
\hline Type B & $172(14.4 \%)$ & $109(14.1 \%)$ & $63(14.6 \%)$ & 0.835 \\
\hline
\end{tabular}

*Statistically significant; DCB: drug-coated balloon; NC: noncompliant; RVD: reference vessel diameter. 
TABLE 4: One-year outcomes.

\begin{tabular}{|c|c|c|c|c|}
\hline Study endpoints & $\begin{array}{l}\text { Overall population } \\
\quad(n=1198)\end{array}$ & $\begin{array}{l}\text { Nondiabetic } \\
\quad(n=768)\end{array}$ & $\begin{array}{l}\text { Diabetic } \\
(n=430)\end{array}$ & $P$ value \\
\hline TLF & $28(2.3 \%)$ & $11(1.4 \%)$ & $17(3.9 \%)$ & $0.006^{*}$ \\
\hline TLR & $13(1 \%)$ & $4(0.5 \%)$ & $9(2 \%)$ & $0.014^{*}$ \\
\hline TVMI & $4(0.3 \%)$ & $1(0.1 \%)$ & $3(0.6 \%)$ & 0.110 \\
\hline Cardiac death & $11(0.9 \%)$ & $6(0.7 \%)$ & $5(1.1 \%)$ & 0.513 \\
\hline MACE & $40(3.3 \%)$ & $21(2.7 \%)$ & $19(4.4 \%)$ & 0.120 \\
\hline Myocardial infarction & $4(0.3 \%)$ & $1(0.1 \%)$ & $3(0.6 \%)$ & 0.110 \\
\hline TVR & $18(1.5 \%)$ & $8(1 \%)$ & $10(2.3 \%)$ & 0.080 \\
\hline All-cause death & $18(1.5 \%)$ & $12(1.5 \%)$ & $6(1.3 \%)$ & 0.820 \\
\hline \multicolumn{5}{|l|}{ Others } \\
\hline $\mathrm{CABG}+\mathrm{PCI}$ & $56(4.6 \%)$ & $33(4.2 \%)$ & $23(5.3 \%)$ & 0.408 \\
\hline Angiogr & $\begin{array}{l}\text { ic follow-up at } 12 \mathrm{mo} \\
\text { Overall population } \\
(n=535)\end{array}$ & $\begin{array}{c}\text { Nondiabetic } \\
\quad(n=349)\end{array}$ & $\begin{array}{l}\text { Diabetic } \\
(n=186)\end{array}$ & $P$ value \\
\hline Reference vessel diameter (mm) & $2.5 \pm 0.7$ & $2.5 \pm 0.8$ & $2.5 \pm 0.2$ & 0.629 \\
\hline Minimum lumen diameter before angioplasty $(\mathrm{mm})$ & $0.35 \pm 1.87$ & $0.39 \pm 2.34$ & $0.28 \pm 0.23$ & 0.315 \\
\hline Minimum lumen diameter after angioplasty $(\mathrm{mm})$ & $2.06 \pm 0.35$ & $2.07 \pm 2.35$ & $2.04 \pm 2.35$ & 0.136 \\
\hline Acute gain & $1.79 \pm 0.41$ & $1.8 \pm 0.41$ & $1.77 \pm 0.42$ & 0.154 \\
\hline $\begin{array}{l}\text { Minimum lumen diameter } 12 \text { months after angioplasty } \\
(\mathrm{mm})\end{array}$ & $1.97 \pm 0.44$ & $2 \pm 0.39$ & $1.89 \pm 0.53$ & $0.007^{*}$ \\
\hline Late lumen loss (mm) & $0.12 \pm 0.30$ & $0.09 \pm 0.25$ & $0.17 \pm 0.37$ & $0.003^{*}$ \\
\hline Binary restenosis, $n(\%)$ & $13(2.4 \%)$ & $4(1.1 \%)$ & $9(4.8 \%)$ & $0.008^{*}$ \\
\hline
\end{tabular}

* Statistically significant. CABG: coronary artery bypass graft; MACE: major adverse cardiac events; PCI: percutaneous coronary intervention; TLF: target lesion failure; TLR: target lesion revascularization; TVMI: target vessel myocardial infarction; TVR: target vessel revascularization.
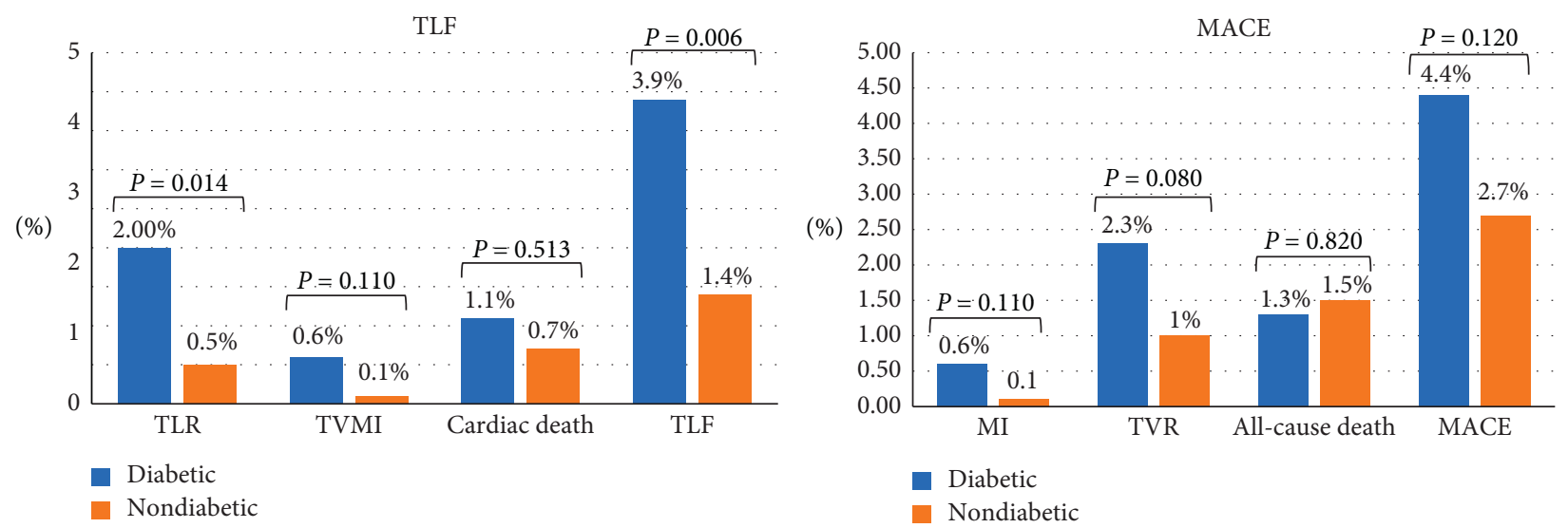

Figure 2: Study endpoints at 12 months. MACE: major adverse cardiac events; MI: myocardial infarction; TLF: target lesion failure; TLR: target lesion revascularization; TVMI: target vessel myocardial infarction; TVR: target vessel revascularization.

month MACE of $4.2 \%$ events rates [32]. The RESTORE SVD China Randomized trial reported 4.3\% TLF events' rates [30]. We observed the lowest TLF (2.3\%) and MACE events' rates (3.3\%) compared to these cited studies. The trend toward lower MACE rates consistently observed with the use of drug-coated balloons in SVD is related to the "leave nothing behind strategy." With the DCB-only angioplasty strategy, it has been observed a postprocedure luminal enlargement; the test using acetylcholine revealed a less pronounced coronary endothelial dysfunction and the absence of acute or late thrombosis [4]. We observed lower MACE rates even in the diabetic group as compared to other studies. Megaly et al. found that DCB had a trend toward lowering TLR in diabetic patients [33]. The BELLO study found that the significant benefit of DCB in the small vessels holds even in the high-risk diabetic patients [29].
The lowest MACE incidence in our study compared to other studies may be partly related to the sample size differences; all the cited studies had a smaller sample size compared to our study. Second, different inclusion criteria were considered; Sinega et al., for instance, enrolled uniquely elderly people who are more likely to have a higher proportion of all the classical risk factors for MACE compared to the general population. DCB angioplasty followed by bailout BMS implantation is known as an independent predictor for poor outcomes [11, 33, 34]. All the bailout stenting was excluded from our study, contrary to the mentioned studies. Besides, appropriate lesion preparation is the key for a successful DCB angioplasty; we were particularly careful to include only procedures where the established recommendations for lesion preparation were observed [38]. Our findings corroborate the 

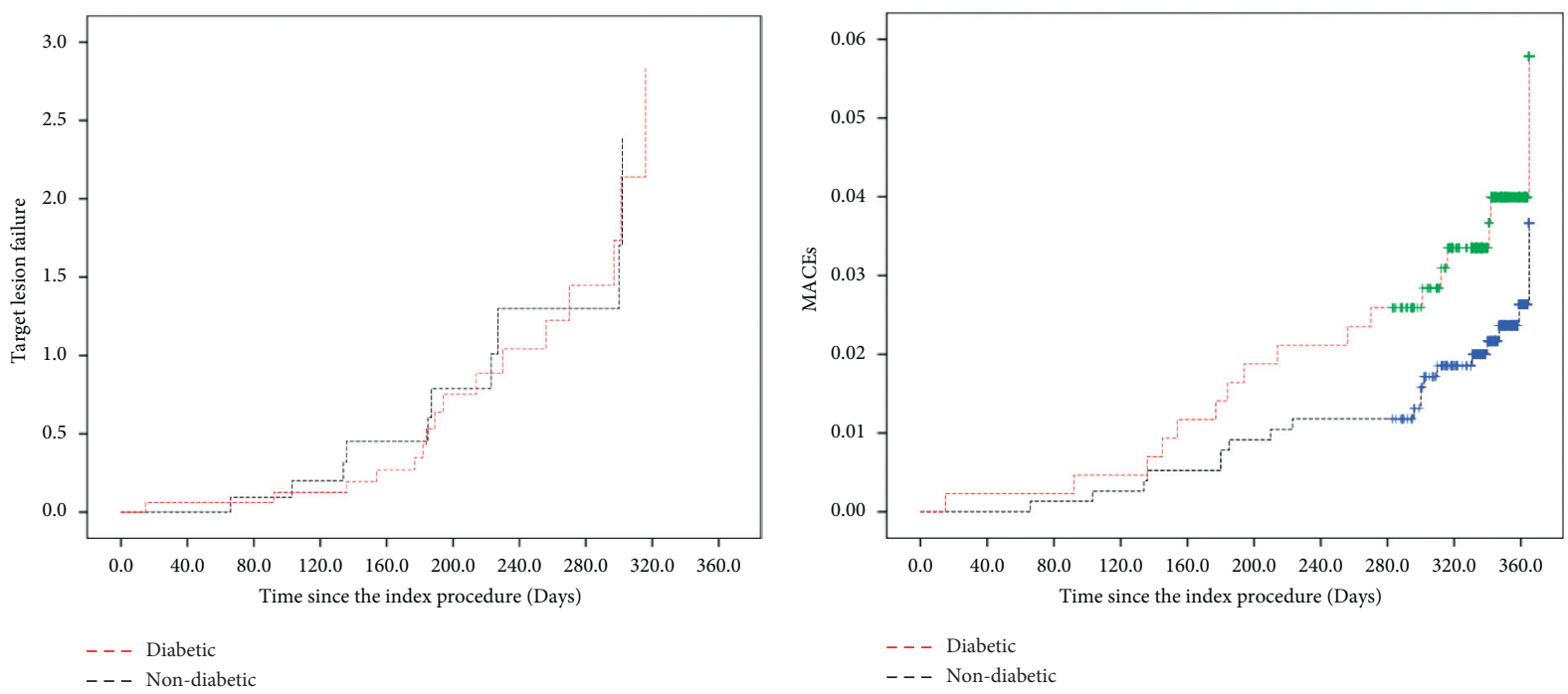

FIgURE 3: Kaplan-Meier curves of the TLF and MACE at 12 months. MACEs: major adverse cardiac events; TLF: target lesion failure.

TABLE 5: Cox proportional hazard multivariate analysis for TLF and TLR.

\begin{tabular}{|c|c|c|c|c|c|}
\hline & & \multirow{2}{*}{$P$ value } & \multirow{2}{*}{$\mathrm{HR}$} & \multicolumn{2}{|c|}{$95 \% \mathrm{CI}$} \\
\hline & & & & Lower & Upper \\
\hline \multirow{7}{*}{ TLR } & Age & 0.841 & 1.006 & 0.951 & 1.064 \\
\hline & Sex & 0.597 & 1.610 & 0.276 & 9.389 \\
\hline & Hypertension & 0.135 & 2.741 & 0.731 & 10.275 \\
\hline & Smoking & 0.082 & 3.144 & 0.865 & 11.423 \\
\hline & Hyperlipidemia & 0.579 & 1.389 & 0.435 & 4.436 \\
\hline & Diabetes & $0.033^{*}$ & 3.698 & 1.112 & 12.298 \\
\hline & Renal insufficiency & 0.955 & 1.064 & 0.124 & 9.144 \\
\hline \multirow{7}{*}{ TLF } & Age & 0.998 & 1.000 & 0.963 & 1.038 \\
\hline & Sex & 0.652 & 1.238 & 0.490 & 3.127 \\
\hline & Hypertension & 0.288 & 1.540 & 0.694 & 3.415 \\
\hline & Smoking & 0.807 & 1.121 & 0.448 & 2.808 \\
\hline & Hyperlipidemia & 0.136 & 1.797 & 0.831 & 3.889 \\
\hline & Diabetes & $0.011^{*}$ & 2.712 & 1.254 & 5.864 \\
\hline & Renal insufficiency & 0.601 & 0.577 & 0.074 & 4.533 \\
\hline
\end{tabular}

Notes. Cox proportional hazard multivariate analysis was performed using age, gender, hypertension, smoking, hyperlipidemia, diabetes, and renal insufficiency. ${ }^{*}$ Statistically significant at $P \leq 0.05$. CI: confidence interval; HR: hazard ratio.

results of the trial by Jalaluddin, which reported significantly higher rates of TLR $(1.4 \%$ vs. $0.6 \% ; P=0.049)$ in the diabetic group [37]. These findings are consistent with the metabolic and hematologic disturbances observed in diabetic patients in contrast with the nondiabetic. Diabetes is associated with accelerated atherosclerosis, widespread endothelial dysfunction resulting in pronounced plaque instability, enhanced platelet aggregation, and clotting response, leading to hypercoagulability $[39,40]$. Compared to the nondiabetic, diabetic patients have greater residual plaque burden throughout the reference segment; the intravascular ultrasound study reported the association between greater plaque burden and edge stenosis with DES and BMS [41-43]. This finding may also be true when $\mathrm{DCB}$ is used. We observed a numerically higher percentage of post-DCB residual stenosis in diabetic patients.

Contrary to our findings, Jalaluddin reported a significantly higher MACE events $(4.3 \%$ vs. $0.6 \% ; P=0.000), \mathrm{MI}$
(2.6\% vs. $0.4 \% ; P=0.002)$ in the diabetic group [37]. A meta-analysis by Sánchez et al. reported that diabetes did not affect the effect of DCB on the TVR [44]. These conflicting results might be related on the one hand to the differences in the inclusion criteria; only patients with $\leq 2.75 \mathrm{~mm}$ vessel diameter were enrolled, and all bailout stenting were excluded from our study. However, Jalaluddin included all patients regardless of the vessel size [37]. Concerning the meta-analysis by Sánchez et al., the heterogeneity of the included trials in terms of the vessel size $(<3 \mathrm{~mm},<2.8 \mathrm{~mm}$, and $\leq 2.75 \mathrm{~mm}$ ) is significant to consider, knowing that the risk of restenosis after PCI is inversely correlated to the treated vessel's size $[12,44,45]$. The enrolled trials included the bailout BMS implantation cases, besides, procedural limitations such as the absence of routine lesion pre dilatation in the PICCOLETO trial, and heterogeneity in the duration of the follow-up among the included trials [44]. 


\section{Conclusion}

The drug-coated balloon-only treatment achieved lower incidence rates of target lesion failure and major adverse cardiac events in de novo small coronary vessel disease based on our data. We reported significantly higher events rates for TLF and individual TLR in the diabetic group than the nondiabetic. However, numerically higher MACE and individual TVR rates, MI, and cardiac death without statistical significance were observed in the diabetic group compared to the nondiabetic. The presence of diabetes is an independent predictor of TLF and TLR, but not of MACE after 12-month follow-up.

5.1. Limitations. First, the absence of angiographic followup for a considerable number of our study population and the short duration of the reported follow-up did not assess the long-term outcomes. Second, because of the small caliber of the treated vessels, ischemic symptoms might have been relatively weaker, consecutively our conclusion might have overestimated the performance of DCB.

\section{Data Availability}

The datasets used and/or analyzed during the current study are available from the corresponding author upon reasonable request.

\section{Ethical Approval}

The authors declare that this work was carried out in accordance with The Code of Ethics of the World Medical Association (Declaration of Helsinki).

\section{Conflicts of Interest}

The authors declare that they have no known conflicts of financial interest or personal relationships that could have appeared to influence the work reported in this study.

\section{Acknowledgments}

The authors acknowledge the help of Clarice Selemani Zanao, Katamu Kalala Frédéric, Edgard Smith, Zhenwen Huang, Sancong Pan, Yanjun Zhou, Zhongsheng, Peisheng Zhang, Luosha Zhao Feifei Zhang, Youyou Du, and Guanghui Liu during data collection, patient's follow-up, and grammar correction. This work was supported by the Medical Science and Technique Research of He'an Province (Provincial and Ministerial Co-Construction Project) (Grants nos. SB201901027 and SB201901010).

\section{References}

[1] A. Solomonica and A. Roguin, "Best percutaneous coronary intervention approach for small caliber coronary arteries," Journal of Thoracic Disease, vol. 8, no. 10, pp. E1268-E1270, 2016.

[2] G. Tarantini, G. Masiero, A. Barioli et al., "Absorb bioresorbable vascular scaffold vs. everolimus-eluting metallic stent in small vessel disease: a propensity matched analysis of
COMPARE II, RAI, and MAASSTAD-ABSORB studies," Catheterization and Cardiovascular Interventions, vol. 92, no. 2, pp. E115-E124, 2018.

[3] E. Puymirat and E. Barbato, "Percutaneous revascularization strategies in small-vessel disease," Annales de Cardiologie et d'Angeiologie, vol. 63, no. 1, pp. 28-31, 2014.

[4] T. Nestelberger and R. Jeger, "Drug-coated balloons for small coronary vessel interventions: a literature review," Interventional Cardiology Review, vol. 14, no. 3, pp. 131-136, 2019.

[5] A. Serra, "Value of drug-coated balloons in small-vessel disease: have they come of age?" JACC: Cardiovascular Interventions, vol. 11, no. 23, pp. 2393-2395, 2018.

[6] D. A. Sinaga, H. H. Ho, T. J. Watson et al., "Drug-coated balloons: a safe and effective alternative to drug-eluting stents in small vessel coronary artery disease: drug-coated balloon angioplasty in small vessel disease," Journal of Interventional Cardiology, vol. 29, no. 5, pp. 454-460, 2016.

[7] H. Mohiaddin, T. D. F. K. Wong, A. Burke-Gaffney, and R. G. Bogle, "Drug-coated balloon-only percutaneous coronary intervention for the treatment of DE Novo coronary artery disease: a systematic review," Cardiology and Therapy, vol. 7, no. 2, pp. 127-149, 2018.

[8] G. Masiero, M. Mojoli, D. Ueshima, and G. Tarantini, "Current concepts on coronary revascularization using BRS in patients with diabetes and small vessels disease," Journal of Thoracic Disease, vol. 9, no. 9, pp. S940-S949, 2017.

[9] F. Picard, S. Doucet, and A. W. Asgar, "Contemporary use of drug-coated balloons in coronary artery disease: where are we now?" Archives of Cardiovascular Diseases, vol. 110, no. 4, pp. 259-272, 2017.

[10] S. Glagov, E. Weisenberg, C. K. Zarins, R. Stankunavicius, and G. J. Kolettis, "Compensatory enlargement of human atherosclerotic coronary arteries," New England Journal of Medicine, vol. 316, no. 22, pp. 1371-1375, 1987.

[11] B. Vaquerizo, F. Miranda-Guardiola, E. Fernández et al., "Treatment of small vessel disease with the paclitaxel drugeluting balloon: 6-month angiographic and 1-year clinical outcomes of the Spanish multicenter registry: placlitaxel eluting balloon for small vessel," Journal of Interventional Cardiology, vol. 28, no. 5, pp. 430-438, 2015.

[12] L. Mauri, E. J. Orav, and R. E. Kuntz, "Late loss in lumen diameter and binary restenosis for drug-eluting stent comparison," Circulation, vol. 111, no. 25, pp. 3435-3442, 2005.

[13] R. Hoffmann, G. S. Mintz, A. D. Pichard, K. M. Kent, L. F. Satler, and M. B. Leon, "Intimal hyperplasia thickness at follow-up is independent of stent size: a serial intravascular ultrasound study," The American Journal of Cardiology, vol. 82, no. 10, pp. 1168-1172, 1998.

[14] X. Wang, W. Lu, X. Wang et al., "Drug-coated balloon angioplasty: predicting outcomes based on different patterns of drug-eluting stent restenosis," The International Journal of Cardiovascular Imaging, vol. 36, no. 2, pp. 171-178, 2020.

[15] B. Stein, W. S. Weintraub, S. S. P. Gebhart et al., "Influence of diabetes mellitus on early and late outcome after percutaneous transluminal coronary angioplasty," Circulation, vol. 91, no. 4, pp. 979-989, 1995.

[16] W. S. Weintraub, B. Stein, A. Kosinski et al., "Outcome of coronary bypass surgery versus coronary angioplasty in diabetic patients with multivessel coronary artery disease," Journal of the American College of Cardiology, vol. 31, no. 1, pp. 10-19, 1998.

[17] R. E. Kuntz, "Importance of considering atherosclerosis progression when choosing a coronary revascularization strategy: the diabetes-percutaneous transluminal coronary 
angioplasty dilemma," Circulation, vol. 99, no. 7, pp. 847-851, 1999.

[18] A. Schömig, C. Schmitt, A. Dibra et al., "One year outcomes with abciximab vs. placebo during percutaneous coronary intervention after pre-treatment with clopidogrel," European Heart Journal, vol. 26, no. 14, pp. 1379-1384, 2005.

[19] M. T. Johnstone and A. Veves, Diabetes and Cardiovascular Disease, Springer, Berlin, Germany, 2014.

[20] C. Ertan, O. Ozeke, M. Gul et al., "Association of prediabetes with diffuse coronary narrowing and small-vessel disease," Journal of Cardiology, vol. 63, no. 1, pp. 29-34, 2014.

[21] B. K. Benjamin, C. Qiu, Z. Han et al., "The association between type-2 diabetes duration and major adverse cardiac events after percutaneous coronary intervention," Journal of Diabetes Research, vol. 2021, Article ID 7580486, 9 pages, 2021.

[22] M. Rosenberg, M. Waliszewski, F. Krackhardt et al., "Drug coated balloon-only strategy in De Novo lesions of large coronary vessels," Journal of Interventional Cardiology, vol. 2019, Article ID 6548696, 8 pages, 2019.

[23] R. V. Jeger, A. Farah, M. A. Ohlow et al., "Drug-coated balloons for small coronary artery disease (BASKET-SMALL 2): an open-label randomised non-inferiority trial," Lancet (London, England), vol. 392, no. 10150, pp. 849-856, 2018.

[24] A. Minacapelli, D. Piraino, D. Buccheri, and B. Cortese, "Drug-coated balloons for the treatment of in-stent restenosis in diabetic patients: a review of currently available scientific data: DCB for the treatment of ISR in diabetic patients," Catheterization and Cardiovascular Interventions, vol. 92, no. 1, pp. E20-E27, 2018.

[25] T. Onishi, Y. Onishi, I. Kobayashi, S. Umezawa, and A. Niwa, "Drug-coated balloon angioplasty for de novo small vessel disease including chronic total occlusion and bifurcation in real-world clinical practice," Cardiovascular Intervention and Therapeutics, vol. 34, no. 2, pp. 139-148, 2019.

[26] American Diabetes Association, "Standards of medical care in diabetes-2014,” Diabetes Care, vol. 37, p. 14, 2014.

[27] K. A. Hicks, K. W. Mahaffey, R. Mehran et al., "2017 cardiovascular and stroke endpoint definitions for clinical trials," Journal of the American College of Cardiology, vol. 71, no. 9, pp. 1021-1034, 2018.

[28] K. Thygesen, J. S. Alpert, H. D. White et al., "Universal definition of myocardial infarction," Circulation, vol. 116, no. 22, pp. 2634-2653, 2007.

[29] A. Latib, A. Colombo, F. Castriota et al., "A randomized multicenter study comparing a paclitaxel drug-eluting balloon with a paclitaxel-eluting stent in small coronary vessels: the BELLO (balloon elution and late loss optimization) study," Journal of the American College of Cardiology, vol. 60, no. 24, pp. 2473-2480, 2012.

[30] Y. Tang, S. Qiao, X. Su et al., "Drug-coated balloon versus drug-eluting stent for small-vessel disease: the restore SVD China randomized trial," JACC: Cardiovascular Interventions, vol. 11, no. 23, pp. 2381-2392, 2018.

[31] M. Unverdorben, F. X. Kleber, H. Heuer et al., "Treatment of small coronary arteries with a paclitaxel-coated balloon catheter," Clinical Research in Cardiology, vol. 99, no. 3, pp. 165-174, 2010.

[32] D. A. Sinaga, H. H. Ho, U. Zeymer et al., "Drug coated balloon angioplasty in elderly patients with small vessel coronary disease," Therapeutic Advances in Cardiovascular Disease, vol. 9, no. 6, pp. 389-396, 2015.

[33] M. Megaly, A. Ali, B. Abraham et al., "Outcomes with drugcoated balloons in percutaneous coronary intervention in diabetic patients," Cardiovascular Revascularization Medicine, vol. 21, no. 1, pp. 78-85, 2020.

[34] C. Yerasi, B. C. Case, B. J. Forrestal et al., "Drug-coated balloon for De Novo coronary artery disease: JACC state-ofthe-art review," Journal of the American College of Cardiology, vol. 75, no. 9, pp. 1061-1073, 2020.

[35] M. Rosenberg, M. Waliszewski, K. Chin et al., "Prospective, large-scale multicenter trial for the use of drug-coated balloons in coronary lesions: the DCB-only all-comers registry," Catheterization and Cardiovascular Interventions, vol. 93, no. 2, pp. 181-188, 2019.

[36] B. Cortese, G. Di Palma, M. G. Guimaraes et al., "Drug-coated balloon versus drug-eluting Stent for small coronary vessel disease: PICCOLETO II randomized clinical trial," JACC: Cardiovascular Interventions, vol. 13, no. 24, pp. 2840-2849, 2020.

[37] M. Jalaluddin, "Treatment of de novo coronary artery lesions with paclitaxel drug coated balloons (DCB) in diabetic and non-diabetic patients," European Heart Journal, vol. 41, no. 2, p. $946,2020$.

[38] F. X. Kleber, H. Rittger, K. Bonaventura et al., "Drug-coated balloons for treatment of coronary artery disease: updated recommendations from a consensus group," Clinical Research in Cardiology, vol. 102, no. 11, pp. 785-797, 2013.

[39] A. S. Weyrich, S. M. Prescott, and G. A. Zimmerman, "Platelets, endothelial cells, inflammatory chemokines, and restenosis: complex signaling in the vascular play book," Circulation, vol. 106, no. 12, pp. 1433-1435, 2002.

[40] M. E. Carr, "Diabetes mellitus: a hypercoagulable state," Journal of Diabetes and Its Complications, vol. 15, no. 1, pp. 44-54, 2001.

[41] K. Sakata, K. Waseda, T. Kume et al., "Impact of diabetes mellitus on vessel response in the drug-eluting stent era: pooled volumetric intravascular ultrasound analyses," Circulation: Cardiovascular Interventions, vol. 5, no. 6, pp. 763-771, 2012.

[42] R. Sakurai, J. Ako, Y. Morino et al., "Predictors of edge stenosis following sirolimus-eluting stent deployment (a quantitative intravascular ultrasound analysis from the SIRIUS trial)," The American Journal of Cardiology, vol. 96, no. 9, pp. 1251-1253, 2005.

[43] J. Liu, A. Maehara, G. S. Mintz et al., "An integrated TAXUS IV, V, and VI intravascular ultrasound analysis of the predictors of edge restenosis after bare metal or paclitaxel-eluting stents," The American Journal of Cardiology, vol. 103, no. 4, pp. 501-506, 2009.

[44] J. S. Sánchez, M. Chiarito, B. Cortese et al., "Drug-coated balloons vs. drug-eluting stents for the treatment of small coronary artery disease: a meta-analysis of randomized trials," Catheterization and Cardiovascular Interventions, vol. 98, pp. 66-75, 2020.

[45] K. D. Buchanan, R. Torguson, T. Rogers et al., "In-stent restenosis of drug-eluting stents compared with a matched group of patients with de novo coronary artery stenosis," The American Journal of Cardiology, vol. 121, no. 12, pp. 15121518, 2018. 